\title{
Animal research: a balancing act
}

\author{
Gains in human health come at the expense of animals in the lab. But denying those gains by hampering animal \\ research could be far costlier.
}

n 2012, animal rights activists pressured passenger airlines to stop air transport of nonhuman primates destined for research labs.

This year, the US Fish and Wildlife Service has recommended that, similarly to wild chimps, all captive chimpanzees be listed as endangered species, thereby drastically limiting their use in experiments, and the US National Institutes of Health (NIH) is decommissioning most of its chimpanzees (http://www.nih.gov/news/health/ jun2013/od-26.htm). In Italy, legislators are seeking to restrict the use of animals in a broad range of research (http://go.nature.com/ NMTb4x). Although the ramifications of these actions differ, changing views of the use of animals in biomedical research need to be balanced with the understanding that society has a continued need for the medical advances derived from animal experiments and of the limitations of existing alternative models (see page 1204).

Biomedical research with animals is fraught with difficulty. Animal models often fail to recapitulate all aspects of a human disease (Nat. Med. 19, 379), are complicated and expensive to use, and require distinct expertise, training, regulatory oversight, and, in some instances, extensive biosecurity controls. Furthermore, animal experiments are subject to ethical concerns not usually raised with in vitro experiments. Oversight and protocol approval bodies endeavor to ensure that animal use in research is ethical, necessary and humane. Yet with the increasing backlash against animal use, the face of biomedical research may change drastically and in uncertain ways.

In June, the NIH announced that it will retire the majority of its chimpanzees from research use and limit its colony available for federally funded research to about 50 animals. To be approved, experiments will need to meet a list of criteria, including that they are necessary for medical improvements to human health and cannot be performed in other animal models or in humans. The decision aims to improve the living conditions of captive chimps in federally funded facilities, given their close genetic relationship to humans, and cites a reduced need for these animals in biomedical research.

In July, the Italian parliament voted in support of a series of amendments to the EU directive 2010/63/EU governing the protection of animals used in research. The amendments would ban the breeding of dogs, cats and nonhuman primates in Italy for research, as well as block studies involving xenotransplantation, the use of animals in drug addiction research and experiments that do not use anesthesia or analgesia and might cause pain to animals (interpreted to include blood draws). The move could cripple biomedical research in the country, particularly in cancer, transplantation, stem cell and addiction research, but also in veterinary and surgical medicine.

The decisions by the NIH and Italy are aligned with the principles of the ' $3 \mathrm{Rs}$ ' - supported in Europe and in the US - to replace, reduce and refine the use of animals in regulatory testing and to develop alternatives to the use of animals in research. However, although the US decision to wind down NIH funded research on chimps affects important areas of biomedicine, including work on hepatitis B and $\mathrm{C}$ viruses, there are alternative in vitro and in vivo research models in place or in development that can at least partially fill the void that chimpanzees will leave. But the far-reaching effect of Italy's amendments restricting animal use cannot be offset by in vitro models, the only alternatives that would not contravene the new laws.

Certainly, new tools and models are needed in all areas of biomedical research to minimize invasive procedures to animals, but the ability to generate in vitro assays that faithfully capture aspects of disease is no trivial goal, and cell culture systems that recapitulate the complexity of higher organisms have yet to be designed. With the ability to engineer organs - such as bladder and kidney - in the lab, and by improving access to human samples, the need for animals in some types of research may eventually be eliminated. But the dynamic interplay between an organ and the body cannot-thus far-be mimicked. Therefore, animals remain crucial to test new vaccines, drugs and surgical methods and to validate noninvasive procedures that eventually can replace their invasive alternatives. Cell culture and unicellular organisms provide invaluable insights into basic biology. Safe and rapid translation of those insights into new therapies for humans-and for animals-requires the humane use of animals.

The 2010/63/EU directive governing 'the protection of animals used for scientific purposes' permits EU member countries to maintain stricter regulations-provided they were in effect on 9 November 2010. Therefore, it is possible that the new, and more extreme, amendments approved by Italy this July may not become law, allowing its research community some measure of hope. And as with nature, research abhors a vacuum, so Italy's loss would no doubt be a gain to other countries' research enterprises, including countries that may have far less regulatory oversight than the EU. The same may be true for research with chimpanzees. We need to weigh carefully whether our restrictive actions serve to help animals or whether by cutting off research we provide an opportunity for those whose work is not so ethically bounded. The cost will be borne by all of us. 